\title{
DELIVERING A TOTAL ENGINEERING EDUCATION
}

\author{
Ian WHITFIELD, Alex DUFFY and Hilary GRIERSON \\ University of Strathclyde, Glasgow, UK
}

\begin{abstract}
The department of Design Manufacturing and Engineering Management (DMEM) at the University of Strathclyde developed the concept of "Delivering Total Engineering" to capture the essence of the teaching, research, and knowledge exchange activity. It is a framework and ethos of the department for its multi-disciplinary and holistic approach throughout the three pillars of design, manufacture and engineering management. This paper defines the Delivering Total Engineering concept from an educational perspective and is used to demonstrate the interconnectivity of the learning and teaching that is needed to innovate in the delivery of capable, competent and confident graduates.
\end{abstract}

\section{Keywords: Delivering total engineering, integrated engineering education}

\section{INTRODUCTION}

Design and its management is at the cusp of engineering activity, it sits at the heart of organised creativity, it is the launch pad for advanced technology and has been identified as a strategic tool for Economic Growth by the European Commission [1]. It is little wonder, therefore, that research into engineering design has attracted so much intellectual effort. However, it has become evident [2] that it is insufficient to focus progress purely on discipline specific or viewpoint specific design issues. Large engineering challenges are multi-disciplinary, and are characterised by scale, complexity and a high degree of involvement of human and organisational factors. Continued commercial successes in engineering ventures rely on the ability to adopt an integrated approach that would support design by taking into account all of these factors. In an attempt to reflect the requirements from a product design engineering education perspective, a practical multi-dimensional framework is presented for connecting the learning and competences, and ultimately to build an understanding of what is required to deliver Total Engineers. To date, there has been no theoretical basis for this concept. Therefore, the work presented in this paper introduces a new direction by attempting to create a holistic design education framework entitled Delivering Total Engineering which is defined as follows:

- Delivering refers to the principles in support of engineering activities: demonstration of the requisite tools, techniques, and methodologies to enable undergraduates to become competent practitioners in preparation for an industrial career.

- Total refers to the scope: to deliver an education that encompasses the entire through-life, lifecycle to lifecycle design, engineering and management. The term "total" is used with the same intention as in Pugh's Total Design or as in Total Quality Management, and is an acknowledgment to Stuart Pugh holding the second Professorial Chair in Strathclyde's Design Division in the early 1990s [3].

- Engineering refers to the context in which DMEM undergraduates are educated and which they progress with their careers: the domain or sector of application. The capability, competence and confidence of DMEM graduates however prepares them for a wider range of opportunities in sectors including finance, service industry, and global IT industries.

It is in the nature of the framework presented here that there is a multiplicity of connections to past, present, and future design practice and education. For example, Pugh's text on Total Design [3] has been a landmark for many engineering design students by formalising and enabling a framework and structure for New Product Development. Total Design is presented as a multi-path framework embedded in a process which is multi-disciplinary, interactive, iterative, directed and explorative. However, looking at "total" design retrospectively, it is clear that there are a number of limitations in the concept. Firstly, it does not attempt to provide an ontological framework for design education. Secondly, it is focused on New Product Development, rather than the breadth of creative engineering problem solving. Finally, it 
does not consider the issues that need to be learned to operate within a global design environment. A major engineering design challenge that has been recognised by industry is captured in the term Systems Engineering, defined as "an interdisciplinary approach and means to enable the realisation of successful systems" [4]. However, while Systems Engineering addresses the multi-dimensional aspects of engineering problems solving, it has been recognised that much remains to be accomplished in the development of interrelationships and processes to cope with modern socio-technological issues [5]. As stated by Hitchins: "The need was envisaged for a systems methodology that was accessible to engineers along with other disciplines from the applied and life sciences, so that the whole process, from addressing the problem to creating the optimum solution, could be understood and pursued with both rigor and expediency" [2]. The creation of the Delivering Total Engineering concept is an attempt to address these requirements from an educational perspective, with particular emphasis placed on the developing the capability for supporting the total dimensionality of design.

This paper presents the outcomes of two preliminary explorations to understand Delivering Total Engineering as an educational support framework. It proposes an ontology by defining a 3D knowledge space that may be used to locate elements of an engineering and product design education. Secondly, it initiates the definition and understanding of Delivering Total Engineering from an educational process perspective applied to the 3D knowledge space.

\section{DELIVERING TOTAL ENGINEERING FRAMEWORK}

Sim and Duffy developed an ontology of generic engineering design activities which reflect a range of skills that are required for practicing engineers that include: abstracting; decomposing; standardising; analysing; decision making; testing/experimenting; and scheduling which were categorised into design definition, design evaluation, and design management activities [6]. In order to deliver a total engineering education, engineers should ideally receive knowledge, understanding and applied learning with respect to tools, techniques and methodologies that correspond to each of these types of activities. This reflects the "Delivering" and first dimension of the total engineering framework.

The design and development of processes, systems, services and products requires a broad consideration of the whole product development process and its management. Pugh's total design illustrates these stages as relating to market analysis, specification, concept design, detail design, and manufacture. In addition, the concept of Design for $\mathrm{X}$ is fundamental in ensuring the designed solution is fit for purpose. Some X's are context specific and depend on the process, system, service or product being developed. Other X's relate to later product development stages including manufacture and assembly, as well as later life phases: operational, maintenance or in-service support, recycle and disposal. In this respect, a through life consideration is required to include circular economy considerations within design. Product design and management learning should be within an education that covers all life phases of the lifecycle of processes, systems, services and products and is therefore the "Total" and second dimension of the Delivering Total Engineering framework.

To conceive the Delivering Total Engineering framework, different aspects of engineering education had to be taken into account. The Engineering Council's UK Standard for Professional Engineering Competence (UK-SPEC) defines competence as "a combination of formal and informal learning, and training and experience" and broadly covers: A) Knowledge and understanding; B) Design and development of processes, systems, services and products; C) Responsibility, management or leadership; D) Communication and inter-personal skills; and E) Professional commitment [7]. Product design engineering education should deliver learning, training and experience with respect to these five competencies. The Engineering Accreditation Board is responsible within UK higher education for decision making with respect to the accreditation of engineering degrees. The Engineering Council lists the output standards and learning outcomes that are required in order to graduate with an accredited engineering degree [8]. The learning outcomes represent the "Engineering" and third dimension of the Delivering Total Engineering framework. These learning outcomes from a bachelor degree accredited for chartered engineering status relate to; science and mathematics (SM); engineering analysis (EA); design (D); economic, legal, social, ethical and environmental context (EL); Engineering practice (P); and, additional general skills (G). Graduating engineers should be able the demonstrate the associated skills to: "solve problems, some of a complex nature, in their chosen engineering discipline" some of which should be through individual and group projects.

To imagine how Delivering Total Engineering would cover these three dimensions, a spatial model was proposed - see Figure 1. 


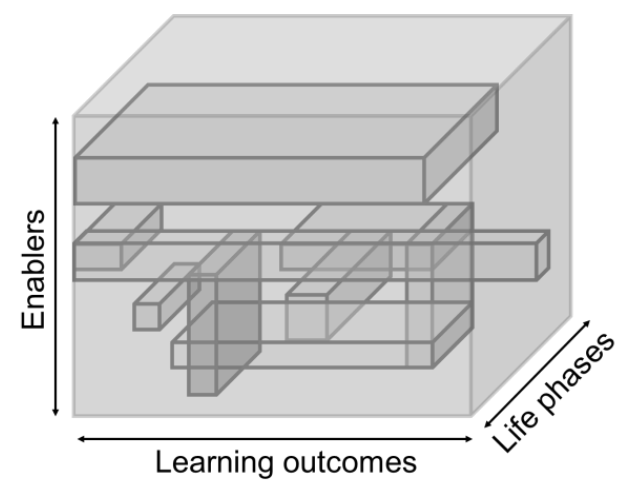

Figure 1 Initial visualisation of Delivering Total Engineering framework.

The rectangular volume shown in the illustration, is intended to encompass the considerations within each of the axes and is a representation of the scope of Delivering Total Engineering. By populating the model with individual modules, delivered within an accredited engineering programme and combine them within a single visualisation, it can have multiple uses. Examples of the intervals associated within the three dimensions of the Delivering Total Engineering framework are detailed within Table 1. This does not reflect a full list of the intervals and is for illustration only. For a complete list of intervals for the Delivering and Engineering dimensions see [6] and [8]. These intervals correspond to a conventional product design perspective and can be tailored in response to process or service design, or with respect to the specific product development demands of an engineering discipline and associated industry.

Table 1. Illustration of intervals for Delivering Total Engineering dimensions.

\begin{tabular}{|l|l|l|}
\hline \multicolumn{1}{|c|}{$\begin{array}{c}\text { Delivering } \\
\text { Dimension }\end{array}$} & \multicolumn{1}{|c|}{ Total Dimension } & \multicolumn{1}{c|}{ Engineering Dimension } \\
\hline Abstracting & Market research & SM1b - Scientific principles and methodology \\
\hline Associating & Concept design & SM2b - Mathematical and statistical methods \\
\hline Composing & $\begin{array}{l}\text { Embodiment } \\
\text { design }\end{array}$ & SM3b - Other engineering disciplines \\
\hline Decomposing & Detailed design & EA1b - Engineering principles \\
\hline Defining & Manufacturing & EA2 - Performance of systems and components \\
\hline Detailing & Assembly & EA3b - Quantitative and computational methods \\
\hline Generating & Operations & EA4b - Integrated or systems approach \\
\hline Standardising & Recycle & D1 - Business, customer and user needs \\
\hline Structuring & Disposal & D2 - Define the problem, identifying any constraints \\
\hline Synthesising & & $\begin{array}{l}\text { D3b - Work with incomplete or uncertain } \\
\text { information }\end{array}$ \\
\hline Analysing & & D4 - Advanced problem-solving skills \\
\hline Decision making & & D5 - Plan and manage the design process \\
\hline Evaluating & & D6 - Communicate their work \\
\hline
\end{tabular}

The undergraduate and postgraduate programmes delivered within DMEM are constructed from modules that develop the skills to become capable, competent and confident engineers. The documentation for these modules provides a module code and title, educational aim, learning outcomes, syllabus, and assessment of the learning outcomes as well as information necessary for the management of the module. These modules can be modelled with respect to the enablers or skills that are developed; stages within the life phases that those skills are learned; the Engineering Council's learning outcomes; and, the competences that are addressed. DM100 which is a first-year design class, covers Competences A, B and D; market research, concept and embodiment design life phases; uses abstracting, composing, decomposing, defining, detailing and standardising; whilst addressing learning outcomes: SM3b, EA4b, D1, D2, D4-D6. This can be illustrated graphically, which for brevity is done using only the "Total" and "Engineering" dimensions. A subset of modules is subsequently used to demonstrate the concept and its use. Figure 1 illustrates eight modules from the UG programme for Competence A: DM100, DM103, DM202, DM205, DM303, DM307, DM400 and DM404. It indicates how these modules individually 
provide learning against each of the stages within the lifecycle as well as against each of the learning outcomes. Only learning outcomes SM1b to EL6 are illustrated however the concept can be extended to all learning outcomes. The main illustration indicates how each of these modules collectively addresses the totality of the learning required. The modules are layered as transparencies within the figure to illustrate overlap.

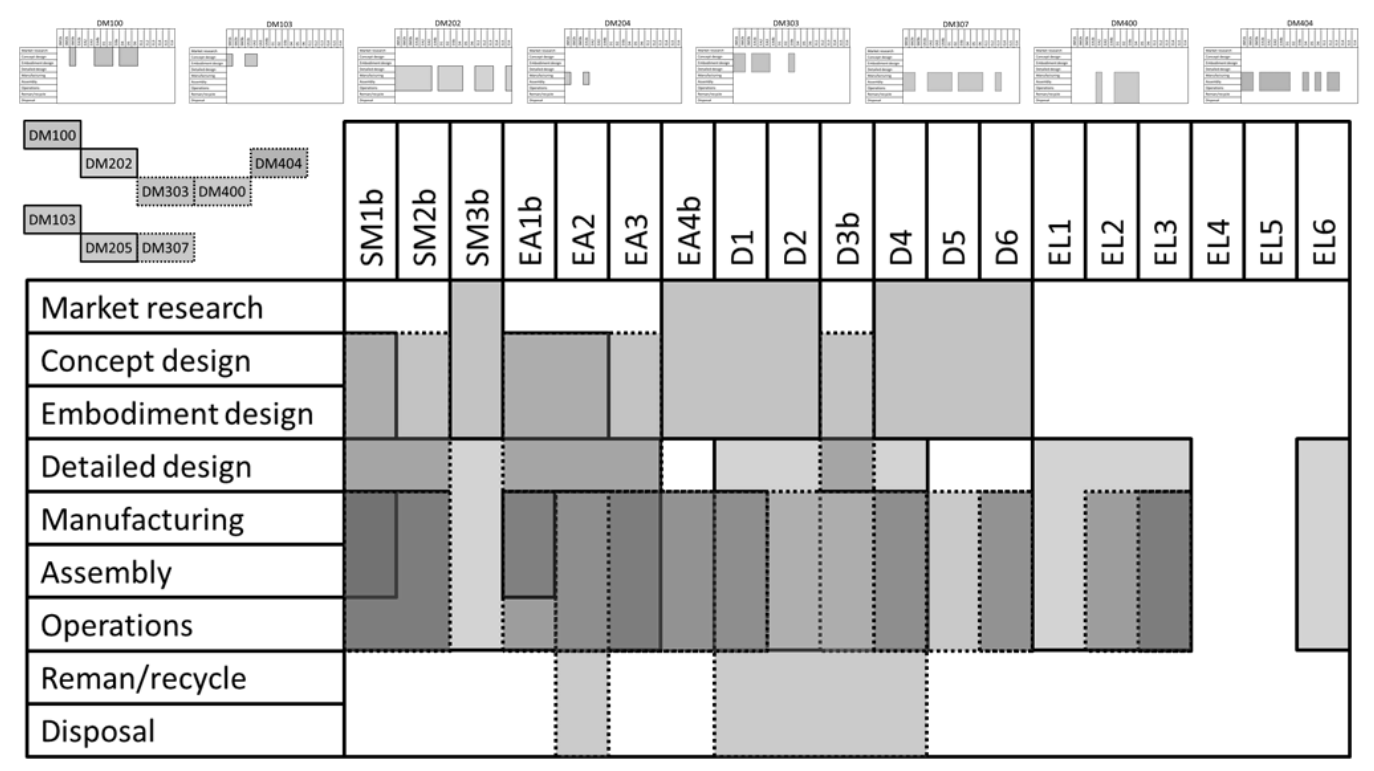

Figure 1. Breakdown of Competence A: Knowledge and understanding

It is clear from Figure 1 that gaps exist where the selected modules do not cover learning outcomes for certain life phases. For example, EL4 (sustainable development) and EL5 (legal requirements) are not considered by the selected modules (but are addressed by other DMEM modules). The gaps provide a useful basis from the perspective of planning a new UG or PG programme. When constructing a new programme, it is important to be able to demonstrate that all of the learning outcomes have been appropriately addressed against the appropriate enablers and life phases. Whilst planning a programme, if a gap were to be apparent, consideration could subsequently be made in terms of whether it would be necessary to develop a new module that addresses the gap, whether the selected modules could be refined, or whether alternative existing modules could be utilised. Due to the layering of modules within Figure 1, it is also possible to see where overlaps exist. These overlaps can provide a useful basis upon which to consider the development of learning from one year to the next, as well as providing a complete illustration of a single programme to ensure thinking is appropriately transferred without being repeated. Figure 1 illustrates the knowledge and understanding is provided within the selected modules which covers market research through to disposal. In addition, the modules used within Figure 1 were selected to illustrate a range of design oriented and manufacturing oriented classes within the earlier years, followed by more advanced design classes in the later years that cover concepts such as Design for Manufacture and Assembly which build upon (and overlap) with the developed learning.

Figure 2 illustrates a selection of modules that were chosen to show the coverage of Competences B to E. In all cases the selected modules were used to provide a representation of the concept, chosen from the product design range of modules, and are not a reflection of the entirety of any individual UG or PG programme delivered by DMEM. For Competence B it can be seen that there is broad coverage across the design-oriented life phases which would be expected for this competence. Competences C, D and E illustrate the influence of including two of the major project orient modules which are individual, and group based. These two modules reflect integrative or capstone projects that aim to demonstrate practical skills with respect to the design and development of processes, systems, services and products. Applied learning is provided within these modules with respect to management and leadership, communication and professional commitment, but not directly with respect to Competence B. Figure 2 also illustrates relative gaps within the recycle and disposal life phases which if it were not appropriately addressed within the existing programmes, could illustrate an opportunity for the future creation of a Sustainable Design programme in the future. 
Competence B: Design and development of processes, systems, services and products

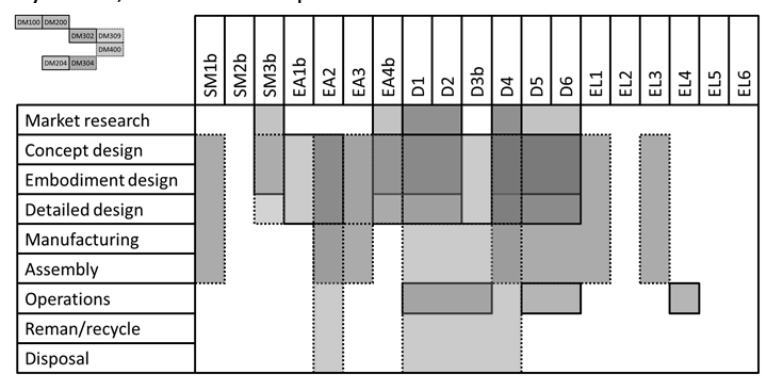

Competence D: Communication and inter-personal skills

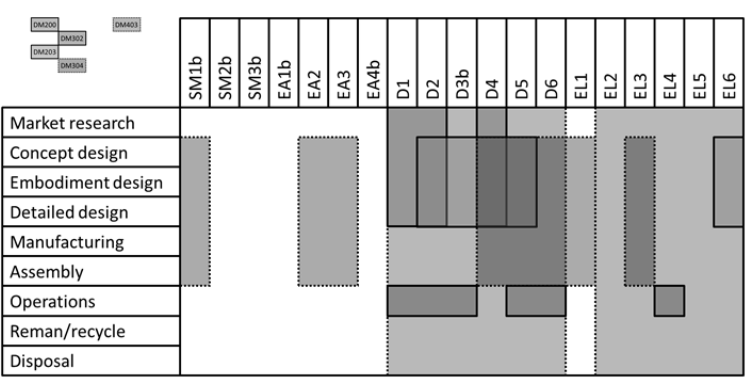

Competence C: Responsibility, management or leadership

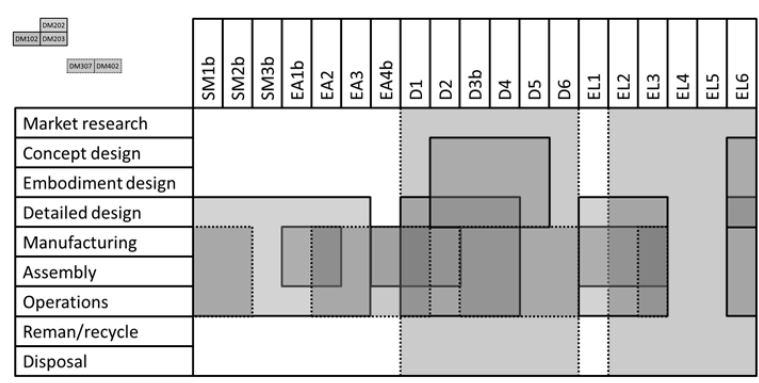

Competence E: Professional commitment

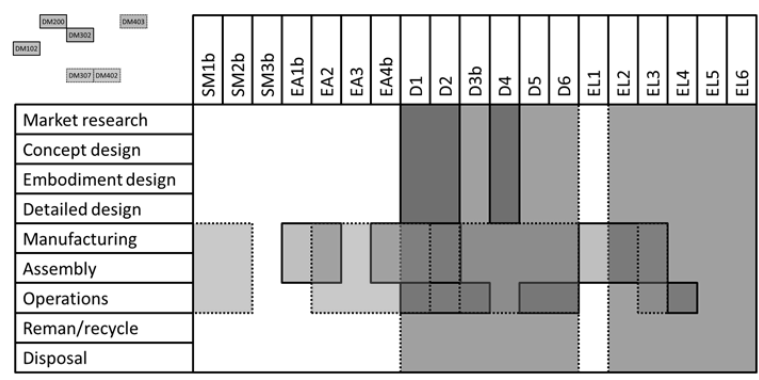

Figure 2. Breakdown of Competences B to $E$

\section{DeLiVERING tOtAL ENGINEERING AS A LEARNING PROCESS}

Design can be defined as the unified and integrated application of engineering sciences, knowledge and skills, to create a future reality which satisfies a perceived need/problem or responds to an opportunity. This future reality could be a new process, system, service or product. A perceived need may be an existing identified problem or a creative opportunity. This definition declares that the smallest unit of engineering design activity starts with a problem or opportunity and terminates with a solution, and we can assume that some kind of transformation takes place between a problem and its solution. From our definition of Delivering Total Engineering the following hypothesis can be postulated: "There exists a problem-directed transform function which produces a solution to a specified problem or opportunity by applying knowledge and understanding of engineering sciences". We can express this hypothesis as: $\tau(\pi, \mathrm{ESc}) \Rightarrow \mathrm{S}$, where: $\tau$ is the problem-directed transform function which should not be taken in the mathematical sense or as a declarative statement in logic; $\pi$ is the identified and specified problem, need or an opportunity; ESc represents the total of available engineering sciences, knowledge and skills; $\mathrm{S}$ is the outcome of an application and is referred to as the solution. In general, the transform function $\tau$ is unknown, but can be thought of as creative process associated with the application of engineering science to search the space of possible solutions. The inverse of the transform function reflects the solution-based learning that can be used to better understand the problem as illustrated in Figure 3. This is simply a graphical representation of the relationships between states and the corresponding transforms which change state. It is not intended to show a flow of information or control.

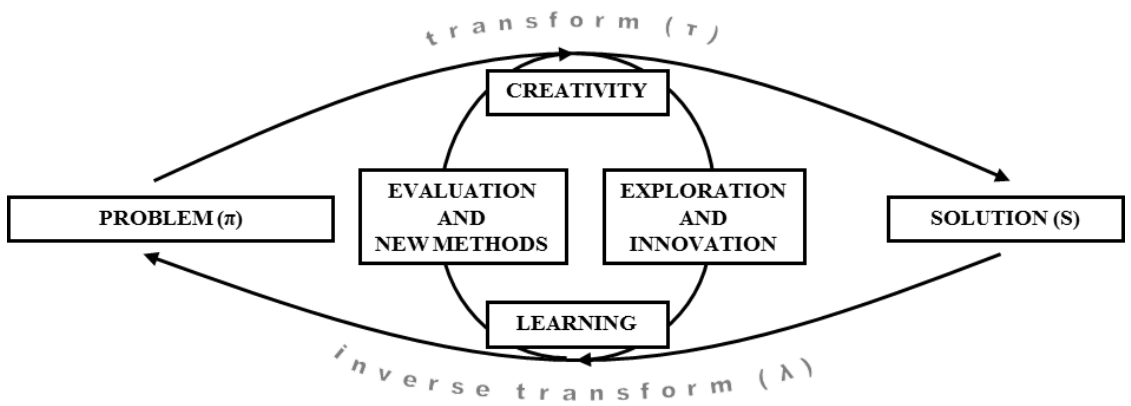

Figure 3. Delivering Total Engineering represented as a learning process 
The process model suggests that having a problem, we arrive at the solution by using the transform function. The concept is iterative in nature, as the Delivering Total Engineering process does not simply come to a finish after the delivery of any single outcome.

These relationships create a retrospective loop of open-ended, creative investigations. Evaluation and new methods, as well as exploration and innovation allows for stretching of experience into learning of new knowledge. The model as further developed in Figure 3 reflects a design architecture model consisting of enabling aspects of each transform (the activities defined by Sim and Duffy [6]), as summarised in Figure 4 below. In such a representation the emphasis is on the problem-solving architecture for enabling tools, techniques, methods, and methodologies.

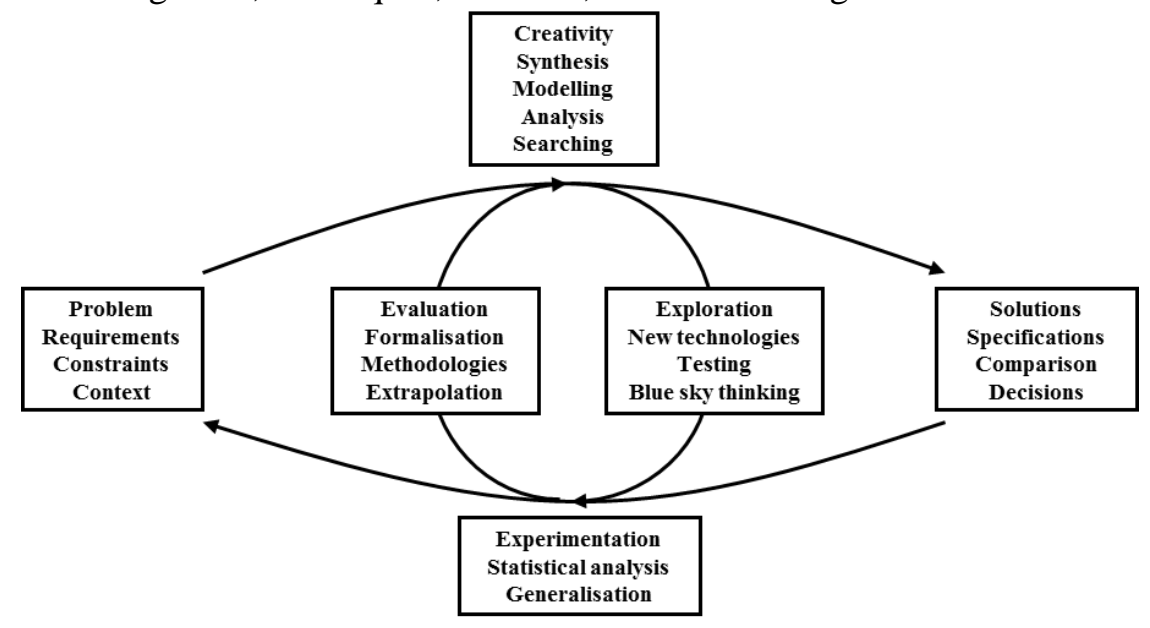

Figure 4. Architectural expansion of the learning process

\section{CONCLUSIONS}

Definition of the concept of Delivering Total Engineering resulted with it being considered as a threedimensional space from an engineering education perspective of principles in support of engineering activities; the scope of through life design; and the learning outcomes required to deliver capable, competent and confident graduates. Individual product design engineering modules, or combinations thereof, can be visualised in this space in order to provide an assessment of the extent to which an educational programme could be considered to deliver total engineering. This assessment can be used in support of the decision making with respect to programme design as well as in support of accreditation. In addition, a complementary process approach to Delivering Total Engineering takes a more analytical view of the subject, defining it as a combination of inter-related states.

The objective of this paper is to provide a basis for further development of the framework for Delivering Total Engineering, and its potential for educational support. More research is needed, particularly to test the applicability of the three defined axes and in selection of intervals. Practical testing of the concept is welcomed, as well as attempts towards its standardisation.

\section{REFERENCES}

[1] Koskinen T. and Thomson M. Design for Growth \& Prosperity, E.D.L. Board, Editor. 2014, European Union. p. 93.

[2] Hitchins D. Systems Methodology, in Conference on Systems Engineering Research, CSER 2005. 2005: Hoboken, New Jersey.

[3] Pugh S. Total Design. 1991, Wokingham: Addison-Wesley.

[4] INCOSE, INCOSE UK SEASON Report 2009, Systems Engineering Annual State of the Nation, U.C.o.t.I.C.o.S. Engineering, Editor. 2009.

[5] Sage A.P. Systems Engineering: Fundamental limits and future prospects. Proceedings of the IEEE, 1981. 69(2): p. 158-166.

[6] Sim S.K. and Duffy A.H.B. Towards an ontology of generic engineering design activities. Research in Engineering Design, 2003. 14(4): p. 200-223.

[7] Engineering Council. UK Standard for Professional Engineering Competence. 2014.

[8] Engineering Council. The Accreditation of Higher Education Programmes. 2014. 